\title{
Experience Based Dynamic Choice: A Revealed Preference Approach *
}

\author{
Amnon Maltz ${ }^{\dagger}$
}

September 28, 2015

\begin{abstract}
We use the revealed preference method to derive a model of dynamic choice where the agent's past experience may influence her current decisions. Our model generalizes the classical individual choice model which is rationalized by utility maximization, and reduces to that model in the absence of experience. As the agent gains experience her utility changes but only in a very restricted fashion. Every period, after an alternative is chosen, the utility of that, and only that alternative, may change while the utility of all other alternatives remains fixed. The model provides a platform on which many behavioral dynamic phenomena may be examined. We utilize it and look into the behavioral implications of bounded memory, status quo bias and variety seeking.
\end{abstract}

Keywords: Experience, Dynamic Choice, Memory, Status Quo Bias, Revealed Preference.

JEL Codes: D11, D83.

${ }^{*}$ I would like to thank Efe Ok for invaluable advice. I would also like to thank Ariel Rubinstein, Andrew Caplin, Paola Manzini, Pietro Ortoleva and Giorgia Romagnoli for very helpful comments.

${ }^{\dagger}$ Department of Economics, University of Haifa. Address: Department of Economics, University of Haifa, Mount Carmel, Haifa, 3498838, Israel. Email: amaltz@econ.haifa.ac.il 


\section{Introduction}

Many empirical studies have established that decision makers are affected by their past experiences. Introspection also suggests that our preferences and choices change as we gain experience in a given market even when other choice related variables remain fixed. These changes may be due to information gathered through experience but other psychological effects, such as boredom, variety seeking or attachment, may also play a role.

One example of experience effects comes from the behavioral economics literature. It has been widely observed that individuals tend to stick to the current state of affairs, a phenomenon known as status quo bias (Samuelson and Zeckhauser 1988). ${ }^{1}$ This phenomenon illustrates one channel through which experience affects choices - that of the last experienced good. Other examples from marketing show that the sequence of past experiences (and not only the most recent one) may also affect choice. Brand loyalty refers to consumers' increasing likelihood of purchasing a good as the number of times it has previously been chosen increases. Variety seekers, on the other hand, try to avoid recently chosen alternatives. ${ }^{2}$ The psychology literature provides further evidence of experience effects. The mere exposure effect, a change in consumer's preferences for a good by repeated exposure to it, was originally observed by Zajonc (1968). In his paper he describes this effect with respect to different types of stimuli such as music and visual images. Later, evidence for this type of behavior, as well as a negative exposure effect, has been found with respect to different types of food. ${ }^{3}$

In this paper we propose a unified framework of dynamic choice which can accommodate all of these behavioral tendencies and more. We view it as a theoretical foundation on which dynamic phenomena may be examined and developed. The model generalizes the classical utility maximization model and allows, at its most basic formulation, flexible behavioral dynamics. After laying down the foundations, we impose more structure on choice and observe in what fashion it narrows down the functional representation.

An agent described by our model maximizes a utility function which changes over time but only in a very restricted fashion. From one period to the next, the utility of the most recently experienced alternative may change while the utility of all the other alternatives remains fixed. Specifically, if the utility of the most recent alternative does not change either, the representation reduces to the standard utility maximization model with no experience effects. More generally, the agent may exhibit various dynamic effects such as learning, attachment or variety seeking. Moreover, the model has the flexibility to allow attachment to some goods and variety seeking with respect to others.

\footnotetext{
${ }^{1}$ Knetsch (1989) as well as Knetsch and Wong (2009) study the phenomenon using every day ordinary goods. See Madrian and Shea (2001) and Choi et al. (2004) for a close examination of 401(K) retirement plans, Johnson and Goldstein (2003) for a study on organ donations and Kempf and Ruenzi (2006) for evidence from mutual fund markets.

${ }^{2}$ See Kahn (1995) for a review as well as Kahn et al. (1986) and Bloemer and Kasper (1995).

${ }^{3}$ Pliner (1982), Crandall (1985) and Stevenson and Yeomans (1995) provide support for the "positive" exposure effect. See Rolls et al. (1981) for evidence of the opposite effect of exposure.
} 
To illustrate our basic representation, suppose an agent is facing a set of three meals in a local restaurant - chicken, beef and pasta. On her first visit to the restaurant she orders chicken, which is the best alternative according to her "experienceless" utility function. That night she feels sick and therefore updates her evaluation of the chicken meal by setting its utility below that of beef and pasta without changing the utilities of those two dishes. According to the model, next time she faces the same menu, the agent will choose either beef or pasta, depending on their utilities, which remain as they were when she first visited the restaurant. In other words, she will choose the same dish she would have chosen on her first visit if chicken was not on the menu that day.

We use the revealed preference approach in a choice domain which is expanded to include not only the feasible choice set but also the decision maker's (DM) past experience. Thus, a choice problem facing our DM takes the form $(S, \sigma)$ where $S$ is the feasible choice set and $\sigma$ is the list of previously chosen alternatives presented in the order in which they were chosen. We allow $\sigma$ to be the empty list, interpreted as a problem in which the DM has no prior experience.

Our basic behavioral traits are captured by two axioms. The first is the weak axiom of revealed preference (WARP) imposed across all problems whose experience structures are identical. Our agent must act rationally in such problems. However, across choice problems with different past experiences, our agent's rationality may fail. Our second axiom imposes structure on the "level of irrationality" allowed across different experience lists. It states that adding a non-feasible alternative, i.e., an alternative that is unavailable for choice in the current choice set, to the existing experience will have no effect on choice. This assumption, which we call reference independence (RI) posits that a reference point has less effect on choice when it must be abandoned.

We utilize our model in order to examine a few well documented behavioral dynamics through the lens of revealed preference. First, we investigate the case of bounded memory and find that if recall is not perfect, i.e., some experience is forgotten with time, the agent is in fact a utility maximizer who ignores experience altogether. This result may be seen as support for the rational choice paradigm at least as far as experience effects are involved. We then study the behavioral postulates which translate into a brand loyal consumer or a variety seeking one. In addition to these formal examinations we provide many examples of dynamic behavior which can be accommodated by our representation. These examples explicitly make use of the expanded choice domain, which includes the agent's past experience, and therefore cannot be described using the standard choice model.

Some existing models concerned with dynamic choice have in fact utilized the functional representation derived in this work. Among those one can find the dynamic utility proposed by Gilboa and Pazgal (2001) as well as Jeuland (1978) used to capture variety seeking and brand loyalty. The literature on switching costs provides another example. It assumes that once a consumer buys a product from a firm, it is harder to get her to buy from a competitor (Klemperer 1987). This behavioral assumption is also accommodated by our model. Thus, the main representation theorem in this work provides axiomatic foundations 
to some existing models of dynamic behavior.

The structure of this article is as follows. Section 2 briefly discusses the related literature. In section 3 we describe the two main axioms and the representation theorem, followed be various examples. Section 4 adds more structure to the model and relates it to different behavioral phenomena. Section 5 concludes and discusses future work. All proofs are given in the appendix.

\section{Related Literature}

Over the past 30 years, economists have been incorporating dynamic utility functions into their models (see Grne-Yanoff and Hansson 2009 for a review and discussion). Among the dynamic concepts studied are learning (Meyer and Sathi 1985, Erdem and Keane 1996), habit formation (Abel 1990, Boldrin et al. 1997, see Rozen 2010 for an axiomatic approach), addiction (Becker and Murphy 1988, see Grossman et al. 1998 for a survey) and variety seeking (Gilboa and Pazgal 2001). ${ }^{4}$ To deal with such "irrational" behavior, typically a utility function is specified to account for the desired dynamic behavior. Our model can accommodate most of these behavioral phenomena and can therefore be viewed as a unified axiomatic foundation for different types of choice dynamics.

Random utility models have also been used to account for dynamics / errors in choices. Among these are the backward looking models and the forward looking models (for a summary see Meyer et al. 1997). With respect to these two types of dynamics the model presented here is backward looking - the agent may be influenced by past experiences but does not take into account how current choices might affect future preferences. ${ }^{5}$ Unlike the random utility models our choice correspondence is deterministic.

The model we propose lies within the realm of choices with frames introduced by Salant and Rubinstein (2008). According to their definition "a frame includes observable information that is irrelevant in the rational assessment of the alternatives, but nonetheless affects choice". The list of past experiences is therefore a specific type of frame; a rational consumer would disregard it but it does affect choice according to our approach.

Our set up has a natural interpretation of the last alternative experienced by the agent as her status quo alternative or endowment. It therefore extends the frameworks studied by Masatlioglu and Ok (2005, 2014), Dean (2008), Ortoleva (2010) and Riella and Teper (2014). In their choice models, the agent chooses from a set of alternatives with or without an initial endowment. In the current model, an observer can see not only the current endowment but all previously experienced goods as well. Due to this enrichment we are able to study additional dynamic behavioral phenomenon which go beyond status quo bias.

\footnotetext{
${ }^{4}$ The literature on the above topics is vast, we only mention a few papers to give a clear idea of the theoretical concepts we are referring to.

${ }^{5}$ In section 5 we discuss how such forward looking behavior may be incorporated into our framework by expanding the choice domain.
} 
Another distinction is that in these models the endowment is always feasible for choice, a restriction suitable for studying status quo bias. In our model we allow for a feasible current endowment as well as an infeasible one. Thus, unlike the status quo bias models, we allow for situations in which an agent is searching for a job after being fired, searching for an apartment after being evicted or simply looking for a cereal in a deli that does not sell the one she previously bought.

\section{Choice with Experience}

In what follows we designate a finite set $X$ to act as the set of mutually exclusive alternatives and assume $|X|>2$. The set $X$ is viewed as the grand alternative space and is kept fixed throughout the exposition. $\Omega_{X}$ is the set of all non-empty subsets of $X$. Let $\mathcal{L}$ be the set of all finite sequences of elements of $X$, including the empty sequence $\emptyset$. Thus a typical element $\sigma \in \mathcal{L}$ is a sequence $\sigma=\left(l_{1}, l_{2}, \ldots l_{n}\right)$ where $n \in \mathbb{N}$ and $l_{i} \in X, \forall i \in\{1, . ., n\}$, or $\sigma=\emptyset$. By a choice problem we mean a list $(S, \sigma)$ where $S \in \Omega_{X}$ and $\sigma \in \mathcal{L}$. The set of all choice problems will be denoted $\mathcal{C}(X)$.

The interpretation of a choice problem $(S, \sigma)$ is that the DM is confronted with the problem of choosing an alternative from the feasible set $S$ having experienced the alternatives in the list $\sigma$ in previous periods. More precisely, when facing a choice problem $(S, \sigma)$ with $\sigma=\left(l_{1}, l_{2}, \ldots l_{n}\right)$, the decision maker has to choose an alternative from the set $S$ given that $n$ periods ago she experienced $l_{1}, n-1$ periods ago she experienced $l_{2}$ and so on. $l_{n}$ is the last alternative she experienced, one period ago, and may be interpreted as her current endowment. ${ }^{6}$ If $\sigma=\emptyset$, the agent chooses without any experience.

By a choice correspondence in the present set up, we mean a function $c: \mathcal{C}(X) \rightarrow$ $\Omega_{X}$, such that

$$
c(S, \sigma) \subseteq S \text { for every }(S, \sigma) \in \mathcal{C}(X) .
$$

A few remarks regarding the general set up:

- A choice correspondence on $\mathcal{C}(X)$ must be non-empty-valued by definition.

- The last alternative in the DM's experience (her endowment), may or may not be available for choice.

- No exogenous constraint is imposed on the relationship between the feasible set and the experience. Specifically, the two may be disjoint, in which case the DM is making a choice from a set with which she had no past experience. ${ }^{7}$

- An experience list may include repetitions, interpreted as alternatives which the agent owned for multiple periods.

\footnotetext{
${ }^{6}$ The periods' length is not specified in the model and should be determined by the context.

${ }^{7}$ We are abusing notation here. Formally, given a choice problem $(S, \sigma)$, this remark refers to $S$ and to the set $\Sigma \equiv\{x \in X \mid x$ is an element in $\sigma\}$.
} 


\subsection{Axioms for choice with experience}

Our general characterization derives from two behavioral traits imposed on the DM. The first is a rationality property familiar from the classical theory of revealed preference. This property warrants that utility maximization takes place for any given list of experience.

Axiom 1 - Weak Axiom of Revealed Preference (WARP). For any $(S, \sigma)$ and $(T, \sigma)$ in $\mathcal{C}(X)$,

$$
c(S, \sigma) \cap T=c(T, \sigma)
$$

provided that $T \subseteq S$ and $c(S, \sigma) \cap T \neq \emptyset$.

This property conditions the behavior of the agent across two choice problems which share the same past experience. It is therefore a reflection of the classical weak axiom of revealed preference to our framework of experience based choice (when $\sigma=\emptyset$ it reduces to the classical formulation of this property). Without any further assumptions, it allows us to think of our DM as if she has a set of utility functions $u_{\sigma}$, one for every list $\sigma \in \mathcal{L}$.

Our next axiom imposes structure on the DM's behavior across choice problems with the same choice set but a different list of experience. It is this behavioral axiom that allows us to derive a link between the aforementioned utility functions. Before we introduce the axiom we need to introduce one small piece of notation.

Notation. For any list $\sigma=\left(l_{1}, l_{2}, . ., l_{n}\right) \in \mathcal{L} \backslash\{\emptyset\}$, and $x \in X$ we denote by $(\sigma, x)$ the concatenated list $\left(l_{1}, l_{2}, . ., l_{n}, x\right)$. For $\sigma=\emptyset$ we write $(x)$ instead of $(\emptyset, x)$.

Axiom 2 - Reference Independence (RI). For any $(S, \sigma)$ and $x \in X$ such that $x \notin S$,

$$
c(S,(\sigma, x))=c(S, \sigma) .
$$

Suppose that in the last period, the agent chose alternative $x$. This period, when she is back to choose another alternative, $x$ is not in the feasible set $S$. Our axiom states that her choice this period will not depend on her last experience with $x$. In other words, her current choice from $S$ will be the same as it would have been a period ago, before experiencing $x$.

$\mathrm{RI}$ is a seemingly strong assumption and we do not wish to claim it is descriptively accurate. It is, in fact, easy to imagine situations in which it may fail. For example, suppose that last week the DM chose a cereal which contains raisins. After tasting it, she realizes that she doesn't like raisins in her cereal. The following week, it is likely that she will refrain from choosing a cereal containing raisins even if the store ran out of the specific one she tried last week. Thus, her recent experience affects her choice even though it is infeasible in the current period. However, there are two main supporting arguments for imposing this axiom, at least as a first step, in our attempt to understand the effects of experience on choice. 
First, there is empirical evidence of it in the context of the exposure effect in food. Stevenson and Yeomans (1995) report that after being exposed to chilli, subjects expressed the same liking for other types of food (and a higher liking for chilli). ${ }^{8}$ There are also other contexts in which it seems acceptable from a descriptive point of view, especially when alternatives are, in some sense, independent of each other. For example, consider a person who is playing a roulette table in some casino and decides to take a walk outside for some fresh air. As she reenters the casino an hour later, she realizes that the table on which she previously played is full. It seems reasonable that her choice of a roulette table from those still available will not be affected by her recent experience.

At a more general level, consider the difference between a choice problem in which the endowment (the most recently experienced alternative) is available for choice and a choice problem in which it is not. Whatever effects the endowment may have on choice, whether it is the well known endowment effect (the phenomenon that the minimum compensation demanded by an agent for a good that she owns is more than the maximum price she is willing to pay for the same good, see Thaler 1980) or any other reference effect, it seems likely they will be mitigated once the endowment is unavailable for choice. The endowment effect for example, may naturally weaken when the individual is aware that she will have to part with the good she currently owns. Other reference effects may be less pronounced due to a lower incentive to compare alternatives to the endowment when it has to be abandoned. We would like to think of our RI axiom as a behavioral postulate that takes this line of reasoning to the extreme. That is, to the point at which all reference effects vanish if the endowment is unavailable for choice.

It is also important to note that imposing RI, though seemingly restrictive, still expands the set of recent models of choice with initial endowment by Masatlioglu and Ok (2005, 2014), Dean (2008), Ortoleva (2010) and Riella and Teper (2014). In all of these models the endowment must be a feasible alternative by construction. Our set up allows for an infeasible endowment, requiring RI to hold in these situations.

\subsection{Characterization of the main model}

Our choice domain is presently quite rich due to the experience being part of the choice problem. Our axioms, on the other hand, seem to be rather restrictive on behavior. Imposing WARP warrants utility maximization taking place every period while RI restrains the manner in which WARP may be violated across periods. Fortunately, the expansion of the domain alongside the imposed behavioral traits strikes a balance, allowing us to connect the utility functions across periods in a meaningful fashion. In particular, the following theorem shows that every period's utility function is strongly related to the utility function that drives choices in the absence of experience.

\footnotetext{
${ }^{8}$ Other experiments testing for the exposure effect do not examine the liking for the other objects that subjects were not exposed to and therefore do not give any information in support of or against our axiom.
} 
Theorem 1 Let $X$ be a non-empty finite set with $|X|>2, \mathcal{L}$ the set of all finite sequences of elements from $X$ and $c$ a choice correspondence on $\mathcal{C}(X)$. Then, $c$ satisfies WARP and $R I$ if and only if, there exist a (utility) function $U: X \rightarrow \mathbb{R}$ and a function $\varphi: \mathcal{L} \rightarrow \mathbb{R}$ such that

$$
c(S, \emptyset)=\underset{x \in S}{\arg \max } U(x)
$$

and

$$
c(S, \sigma)=\underset{x \in S}{\arg \max } U_{\sigma}(x)
$$

where

$$
U_{\sigma}(x)=U(x)+\sum_{i=1}^{k} \varphi\left(\left(l_{1}, l_{2}, \ldots, l_{i}\right)\right) \mathbb{1}_{l_{i}}(x)
$$

for every $S \in \Omega_{X}$ and list $\sigma=\left(l_{1}, \ldots, l_{k}\right) \in \mathcal{L} \backslash\{\emptyset\}, k \in \mathbb{N}$.

This result identifies a representation of experience based choice. Our agent starts off as a classic utility maximizer when she has no experience. As she gains experience she remains a utility maximizer but her utility function may change. This much is simply an interpretation of WARP. The RI axiom narrows down the manner in which the utility function is allowed to change. Experiencing an option leads to a change in the utility of that option alone without affecting the utility of any other option.

To illustrate, suppose we examine our DM's choices of cars in two periods. At her first visit to the dealer she has in mind a preference relation over cars expressed by a utility function $U$. She chooses the best available car according to $U$, say a Buick. Next year, when she returns to the dealer to buy a new car, she has a new "experienced utility function" in mind. The utility values of all cars except Buick are exactly as they were last year. The utility of Buick may have increased, decreased or remained the same according to the past year's experience. She buys the best car according to this new utility function.

The example illustrates that this model is best suited to the interpretation of elements of $X$ belonging to a specific category of goods (such as cars, cereals, hotels, etc.). Although no formal reason holds us from treating the grand set as, say, consumption bundles, the interpretation of the RI axiom as well as the representation theorem seem less plausible in these contexts. Thus, the foundational ground of this model with respect to habit formation or addiction is better suited to goods within a specific product market rather than for aggregate levels of consumption. Brand loyalty, variety seeking and learning are naturally suitable to this framework without any restrictions as they are behavioral phenomena typically observed within a specific product market.

\subsection{Examples}

Example 1. (Experience Independent Choice) A decision maker who is completely unaffected by experience is captured by the choice model of Theorem 1. Indeed, by setting 
$\varphi(L)=0, \quad \forall L \in \mathcal{L}$, the representation becomes

$$
c(S, \sigma)=\underset{x \in S}{\arg \max } U(x) \text { for all }(S, \sigma) \in \mathcal{C}(X),
$$

thus reducing to the classic static model of choice.

Example 2. (Extreme Status Quo Bias) Consider a decision maker whose choice behavior is vulnerable to the status quo bias at the highest level, meaning she never leaves her current endowment as long as it is feasible for choice. Such an agent may be described using the above model by setting $\varphi(L)=" \infty ", \forall L \in \mathcal{L}{ }^{9}$

In passing we would like to note that the first two examples could also be captured by the status quo bias model of Masatlioglu and Ok (2014). Their model describes an agent who is a constrained maximizer. The agent maximizes her utility given a constraint set which is induced by her endowment. Thus their model captures the classical agent of Example 1 if she considers all available options from any endowment's perspective. The agent of Example 2 is described by their model by assuming the decision maker does not consider any alternative except for the one she is endowed with.

Notice that the psychological procedures leading to extreme status quo bias are completely different in the two models. In the status quo bias model it is due to the extremely narrow constraint set (the singleton set containing only the endowment) while the endowment's utility remains the same. In our model, the endowment simply seems the best alternative after the agent sets her new rankings in mind based on her last experience. The behavioral effects enter through completely different psychological channels, yet the two models cannot be distinguished based on choice alone in the case of extreme status quo bias.

We now turn to examples which are unique for our extended model of choice as they make use of the sequential structure of the list of experience.

Example 3. (Extreme Variety Seeking) The model derived in Theorem 1 is suitable for situations in which a DM constantly moves away from her endowment even when it is available for choice. Such behavior can be captured by setting $\varphi(L)="-\infty ", \forall L \in \mathcal{L} .{ }^{10}$ Such an agent will always switch away from her endowment as long as there is another available option. We can think of this sort of behavior as expressing extreme boredom with the status quo alternative, the exact opposite behavior to the one expressed in Example 2.

\footnotetext{
${ }^{9}$ By " $\infty$ " we mean a value which when added to the utility of the alternative last experienced brings its utility to the highest overall. For example, we may use the difference between the utilities of the highest ranked and lowest ranked alternatives and add 1 to that difference. Since $X$ is finite and lists are finite sequences we can obviously do so formally for every list. In order to keep notation simple and intuitive we simply denote this value by " $\infty$ ".

${ }^{10}$ Similar to our notation in example 2 , when we write " $-\infty$ " we mean a value which ensures the last experienced alternative's utility is lower than that of all other alternatives.
} 
Example 4. (Cycles) Example 3 immediately paves the way for agents to exhibit dynamic cycles. Consider an agent with the choice behavior described in Example 3. Suppose in the first period she chooses alternative $x$. In period 2 she faces the choice between $x$ and $y$. Due to her extreme boredom she switches away from $x$ and chooses $y$. Suppose that in the third period she is once again asked to choose between these two alternatives. After experiencing $y$ she has now grown bored of it, thus choosing $x$ once again and creating a dynamic three period cycle.

Example 5. (Learning) Imagine an individual who originally has a prior over the quality of the goods and learns their true quality only after experiencing them. Once experienced, her valuation for a good remains fixed. The model would be adapted to this type of behavior by setting $\varphi\left(l_{1}, . ., l_{n}\right)=0$ if $l_{n} \in\left\{l_{1}, \ldots, l_{n-1}\right\}$. This agent may be thought of as a fully rational agent which simply updates her preferences through experience. Once updating is complete (after experiencing all elements in $X$ ), she has a static utility function.

Example 6. (Gradual Addiction / Gradual Boredom) Suppose we choose $\varphi(L)=1, \forall L \in$ $\mathcal{L}$. This specification describes an agent who becomes more and more biased towards her endowment the longer she holds on to it. However, she may well switch away from it if an alternative with a higher utility is available. The longer this agent stays with her endowment the smaller the set of alternatives for which she will opt to switch. This is an example of addictive behavior and is also in line with the mere exposure effect found in the psychology literature. ${ }^{11}$ By setting $\varphi(L)=-1, \forall L \in \mathcal{L}$, we obtain gradual boredom the agent will switch away from her endowment at some period but not necessarily every period.

Example 7. (Sudden Extreme Satiation) Suppose an agent consumed a specific type of food for a long period of time and then suddenly could not bear to taste it anymore. This may happen to individuals who had a very limited variety of food due to extreme poverty or shortage in supply, for example during wartime. At some stage, after a long period of no nutritional variety they developed strong disliking for the type of food which was available. Our model can be adjusted to capture such behavior by assuming $\varphi(\underbrace{x, \ldots, x}_{k})=0$ for some $k \in \mathbb{N}$ and $\varphi(\underbrace{x, \ldots, x}_{k+i})="-\infty$ " for all $i \in \mathbb{N}$.

Example 8. ("Childish Preferences") Suppose $\varphi: \mathcal{L} \rightarrow \mathbb{R}$ takes the following functional form:

$$
\varphi\left(l_{1}, . ., l_{n}\right)=\left\{\begin{array}{cl}
1 & \text { if } l_{n} \notin\left\{l_{1}, \ldots, l_{n-1}\right\} \\
-10 & \text { otherwise }
\end{array}\right.
$$

\footnotetext{
${ }^{11} \mathrm{We}$ axiomatize this gradual addictive behavior in section 4 .
} 
Under this formulation the agent enjoys a utility boost only if she experiences alternatives which she never experienced before. Once experienced, the agent gets bored of that alternative resulting in a utility decrease. This seems to describe young children's attitude towards toys - a new toy is highly valued and played with for one or two days after which it is placed in a box not to be looked at for years..

Example 9. (Opening up for change) Imagine an agent which is extremely status quo biased in the sense of Example 2. Suppose this agent buys the same sesame bagel every morning for 3 years. One morning, there are no more sesame bagels in the shop. The agent chooses her second favorite alternative which is available - a poppy bagel (which she actually never tried before). She realizes that she likes this bagel more than than the sesame bagel. Due to this realization, the boost that her chosen alternatives receive from now on is much lower (since she understood her early bias prevented her from experiencing delicious bagels). Formally this agent can be described by:

$$
\begin{cases}\varphi(\underbrace{x, \ldots, x}_{i})=" \infty ", & \text { for all } x \in X \text { and all } i \in \mathbb{N} \\ \varphi(\underbrace{x, \ldots, x}_{i}, y)=" \infty ", & \text { for any } i \geq 2 \text { and for any } y \neq x \\ \varphi(\underbrace{x, \ldots, x}_{i}, y, z)=1, & \text { for all } y \neq x \text { and for all } z \in X\end{cases}
$$

\section{Structural Experience Based Choice}

So far, we developed a flexible model of experience based choice where no structure was imposed on the change in utility of the current endowment. In this section we add behavioral assumptions which give rise to a more structural dynamic utility model by narrowing down the range of $\varphi$.

\subsection{Memory}

An agent who abides to the choice procedure described in Theorem 1 has dynamic preferences which depend on arbitrarily long past experiences. Such an agent's current utility function may be affected by alternatives experienced many periods ago. In most contexts this seems too strong of a requirement. It is much more likely that recent experiences have a stronger influence on current decisions than old ones. Here we consider the case of bounded memory in which options experienced a long time ago are simply forgotten. Surprisingly, we find that if the agent's memory is limited, her preferences are completely independent of all past experiences, including those she does recall. Moreover, this result is independent of how limited the agent's memory is: as long as memory is imperfect, there is actually no memory at all. We will now state this formally. 
Suppose our agent is able to remember a fixed number of past experiences but not more. For example, a memory-10 agent can remember all her past history as long as it is shorter than 10 periods. Once her accumulated experience comprises more than 10 periods, she is able to recall only the last 10 . The following axiom is the behavioral trait that conveys this exact notion of limited memory.

Axiom 3 - Memory- $K$. Let $K \in \mathbb{N}$. For any list $\sigma \in \mathcal{L} \backslash\{\emptyset\}, \sigma=\left(l_{1}, l_{2}, \ldots, l_{n}\right)$, with $n>K$ :

$$
c\left(S,\left(l_{1}, l_{2}, \ldots, l_{n}\right)\right)=c\left(S,\left(l_{2}, \ldots, l_{n}\right)\right)=\ldots=c\left(S,\left(l_{n-K+1}, \ldots, l_{n}\right)\right) .
$$

It is, of course, possible to imagine situations in which the axiom is violated. For example, many individuals never forget their first day in school. First time experiences in general may be extremely meaningful and leave an unforgettable impression on individuals. Still, we believe that our memory axiom applies to most circumstances, if not precisely, then at least in its conceptual flavor. Innocent on its own though it may be, adding limited memory to the two other axioms gives rise to no other than a completely standard utility maximizer.

Proposition 1 Let $X, \mathcal{L}$ and $c$ be as in Theorem 1. Then, $c$ satisfies WARP, RI and Memory-K if and only if, there exist a (utility) function $U: X \rightarrow \mathbb{R}$ such that

$$
c(S, \sigma)=\underset{x \in S}{\arg \max } U(x)
$$

for every $S \in \Omega_{X}$ and list $\sigma \in \mathcal{L}$.

Proposition 1 states that an agent satisfying our first three axioms is completely unaffected by her experience. She makes her choices by simply evaluating options according to her "experienceless" utility function. In some sense this result may be viewed as a manifestation of rational choice. After all, RI may indeed be a strong axiom but, as we argued, is natural in some contexts and is a reasonable approximation in others, while Memory-K is a very natural trait to describe limited memory capacity. The fact that the combination of the two with WARP gives rise to utility maximization is support of the rational choice paradigm, at least as far as deviations from it due to past experiences are concerned.

\subsection{Status Quo Bias / Brand Loyalty}

Our set up is a natural one for considering agents that are vulnerable to the status quo bias phenomenon or exhibit brand loyal type of behavior. Example 2 describes a very extreme case of the bias which may be captured by our model. Example 6 highlights the same phenomenon but in a more gradual manner. The tendency to stick to the current endowment, and the possible dynamics of such a tendency, translate in a very natural way to a restriction on $\varphi$. In this subsection we impose two axioms which are natural extensions 
to the axioms described in the status quo bias model of Masatlioglu and Ok (2014) to our set-up. The added structure obtained by these axioms follows.

Axiom 4 - Weak Status Quo Bias (WSQB). For any $x, y \in X$ and $\sigma \in \mathcal{L}$,

$$
y \in c(\{x, y\},(\sigma, x)) \Rightarrow y \in c(\{x, y\}, \sigma)
$$

and

$$
y \in c(\{x, y\}, \sigma) \Rightarrow y \in c(\{x, y\},(\sigma, y))
$$

This is a very natural postulate to impose on a decision maker who may be vulnerable to the status quo bias phenomenon (at least in the weak sense). Put simply, it states that serving as an endowment can only enhance an alternative's possibility of being chosen over another. More specifically, if $y$ is chosen over $x$ when $x$ is the last experienced good then it must also be chosen over $x$ prior to that last experience. The second part is similar - if $y$ is revealed preferred to $x$ given an experience list $\sigma$ then it must also be revealed preferred to it when $y$ is added to the experience list. ${ }^{12}$ It is important to note that the axiom is stated in a "weak" fashion which allows the decision maker to exhibit, or not to exhibit status quo bias, depending on the alternatives at hand. Specifically, a rational agent who completely ignores her experience will satisfy this axiom.

Proposition 2 Let $X, \mathcal{L}$ and $c$ be as in Theorem 1. Then, c satisfies WARP, RI and $W S Q B$ if, and only if, the representation of Theorem 1 holds and $\varphi(\sigma) \geq 0$, for all $\sigma \in \mathcal{L}$.

As in the more general case, this agent only changes the utility of her recently experienced alternative. The additional structure lies in the direction of the change. An agent satisfying WARP, RI and WSQB always has a weakly better evaluation of a good after it has been experienced. If she experiences the same good over and over again, every experience will only add (weakly) to its value leading to a type of brand loyalty. Such an agent can be thought of as having reinforced status quo biased behavior through the channel of a "utility boost". ${ }^{13}$ Due to our domain expansion, this model allows the status quo bias to become "stronger" as time goes by, a phenomenon which is impossible to describe in the frameworks suggested to study the status quo bias phenomenon (Masatlioglu and Ok 2005, 2014, Dean 2008, Ortoleva 2010 and Riella and Teper 2014).

An agent satisfying WSQB updates her utility in a weakly positive fashion. If one would like to obtain strictly positive updating, the following stronger version of the axiom is required.

\section{Axiom 5 - Medium Status Quo Bias (MSQB).}

$$
x \in c(\{x, y\}, \sigma) \Rightarrow\{x\}=c(\{x, y\},(\sigma, x)) .
$$

\footnotetext{
${ }^{12}$ For a more elaborate discussion of this axiom see Masatlioglu and Ok (2014).

${ }^{13}$ For a different axiomatic approach leading to a utility boost see Masatlioglu and Ok (2005).
} 
MSQB states that if an alternative $x$ is chosen over an alternative $y$, given any experience $\sigma$, adding $x$ to the experience will make $x$ strictly preferred to $y$ according to the revealed preferences. ${ }^{14}$ As opposed to WSQB, this axiom rules out that our agent is a standard utility maximizer. As MSQB is more demanding a requirement than WSQB, imposing it allows us to obtain even more structure on the function $\varphi$.

Proposition 3 Let $X, \mathcal{L}$ and $c$ be as in Theorem 1. Then, c satisfies WARP, RI and $M S Q B$ if, and only if, the representation of Theorem 1 holds and $\varphi(\sigma)>0$, for all $\sigma \in \mathcal{L}$.

Unlike the case of Proposition 2, this agent will never maintain the same utility value for an experienced good. Rather, experiencing a good always makes it strictly better in the utility sense.

Notice that we can easily impose analogous axioms to WSQB and MSQB which lead to weak or strict types of variety-seeking behavior. For example, we can impose that after experiencing an alternative, it is never chosen from any non-singleton choice set. This will naturally lead to the agent having $\varphi="-\infty$ " as in Example 3. The analysis is similar to the status quo biased behavior discussed above and is therefore omitted.

\section{Discussion and Future Work}

In this work we approach experience based dynamic choice via revealed preferences. We expand the choice domain to allow for past experiences to affect choices. Our agent starts off with an "experienceless" utility function and every period updates the utility of the most recently experienced alternative, while the utility of all other alternatives remains fixed. We view this exercise as setting a platform on which different dynamic behavioral phenomena may be studied in a unified manner. Utilizing it, we examine the axiomatic foundations as well as behavioral implications of bounded memory, status quo bias and variety seeking.

We view this model as a first step towards a more complete theory of choice with experience. Our main axiom, reference independence, imposes a lot of structure on the dynamic nature of choices. We believe it would be interesting to weaken this axiom and allow the endowment to affect choices even when it is not feasible. Another axiom that one might be interested in weakening is that of bounded memory. Though the result of adding this axiom to our model is surprising in that it eliminates all experience effects, it may be more realistic to assume that the influence of experience gradually diminishes with time. In terms of the representation, we conjecture that this will make $\varphi$ a function not only of the list but also of the time elapsed since the last alternative on the list was experienced.

\footnotetext{
${ }^{14}$ MSQB is a weaker axiom than the adapted version of SSQB used in Masatlioglu and Ok (2014). That is also the reason we dub this axiom Medium Status Quo Bias rather than Strong Status Quo Bias which is already used in their paper.
} 
Forward looking agents is another route to take using this set up. One may consider further expanding the choice domain to include not only the past experience but also the number of periods ahead of the decision maker. Axioms could then be imposed across time horizons to contain the possible changes of utilities along this dimension. Finally, one may be interested in imposing more structure on the model in addition to the two basic axioms as we did in Section 4 to highlight other experience related behavioral phenomena.

\section{Appendix}

Proof of Theroem 1. The "if" part of the theorem is trivial. We show the "only if" part. Let $c$ be a choice correspondence on $\mathcal{C}(X)$ that satisfies WARP and RI. Define the binary relation $\succeq$ on $X$ by

$$
y \succeq x \text { if and only if } y \in c(\{x, y\}, \emptyset) .
$$

A standard argument, based on WARP, shows that $\succeq$ is a complete preorder on $X$. In addition, WARP implies

$$
c(S, \emptyset)=\{\omega \in S: \omega \succeq x \text { for all } x \in S\} .
$$

Given that $X$ is finite, we can find a utility function $u$ that represents $\succeq$. Therefore:

$$
c(S, \emptyset)=\underset{x \in S}{\arg \max } u(x) \text { for every } S \in \Omega_{X} .
$$

We now move on to the second part of the representation. $\varphi: \mathcal{L} \rightarrow \mathbb{R}$ and $u_{\sigma}: X \rightarrow \mathbb{R}$ will be defined recursively. For any $x \in X$ define: ${ }^{15}$

$$
\begin{gathered}
A_{0}:=\{(z, y) \in X \times X: u(z) \neq u(y)\} \\
\delta_{0}:=\left\{\begin{array}{cc}
1 & \text { if } A_{0}=\emptyset \\
\min _{(z, y) \in A_{0}}|u(z)-u(y)| / 2 & \text { otherwise }
\end{array}\right. \\
\succ_{(x)}^{x}:=\{\omega \in X: \omega \neq x,\{\omega\}=c(\{\omega, x\},(x))\} \\
\sim_{(x)}^{x}:=\{\omega \in X: \omega \neq x,\{\omega, x\}=c(\{\omega, x\},(x))\}
\end{gathered}
$$

Using the above, we define:

$$
\varphi(x)= \begin{cases}u(y)-u(x) \text { for } y \in \sim_{(x)}^{x} & \text { if } \exists z \neq x \text { s.t. }\{x, z\}=c(\{x, z\},(x)) \\ \max _{z \in X}\left[u(z)+\delta_{0}-u(x)\right] & \text { if }\{x\}=c(\{x, z\},(x)) \forall z \in X \\ \min _{z \in \succ_{(x)}^{x}}\left[u(z)-\delta_{0}-u(x)\right] & \text { otherwise }\end{cases}
$$

\footnotetext{
${ }^{15}$ We abuse notation and write $\varphi(x)$ instead of $\varphi((x))$.
} 
Claim. $\varphi$ is well defined.

Proof of Claim. Let $y_{1} \neq y_{2},\left\{y_{1}, y_{2}\right\} \subseteq \sim_{(x)}^{x}$. If $\{x\}=c\left(\left\{y_{1}, y_{2}, x\right\},(x)\right)$ then WARP implies $\{x\}=c\left(\left\{x, y_{1}\right\},(x)\right)$, contradicting $y_{1} \in \sim_{(x)}^{x}$. By the same argument it follows that $\left\{y_{i}\right\} \neq c\left(\left\{y_{1}, y_{2}, x\right\},(x)\right)$ for $i \in\{1,2\}$.

Similarly, WARP implies $\left\{y_{i}, x\right\} \neq c\left(\left\{y_{1}, y_{2}, x\right\},(x)\right)$ for $i \in\{1,2\}$. Thus, $\left\{y_{1}, y_{2}\right\} \subseteq$ $c\left(\left\{y_{1}, y_{2}, x\right\},(x)\right)$. By WARP and RI:

$$
\left\{y_{1}, y_{2}\right\}=c\left(\left\{y_{1}, y_{2}, x\right\},(x)\right) \cap\left\{y_{1}, y_{2}\right\}=c\left(\left\{y_{1}, y_{2}\right\},(x)\right)=c\left(\left\{y_{1}, y_{2}\right\}, \emptyset\right)
$$

It follows that $u\left(y_{1}\right)=u\left(y_{2}\right)$ and $\varphi$ is well defined.

Now define $u_{(x)}: X \rightarrow \mathbb{R}$ as:

$$
u_{(x)}(y):=u(y)+\varphi(x) \mathbb{1}_{x}(y), \text { for every } y \in X .
$$

We now turn to recursive part of the definitions. Take any $n \in \mathbb{N}$. Suppose we already defined $\varphi: \mathcal{L} \rightarrow \mathbb{R}, u_{\sigma}: X \rightarrow \mathbb{R}$ for all lists with length smaller or equal to $n$. Take any $\sigma \in \mathcal{L}$ with length smaller or equal to $n$ and any $x \in X$. Define: ${ }^{16}$

$$
\begin{gathered}
A_{\sigma}:=\left\{(z, y) \in X \times X: u_{\sigma}(z) \neq u_{\sigma}(y)\right\} \\
\delta_{\sigma}:=\left\{\begin{array}{cc}
1 & \text { if } A_{\sigma}=\emptyset \\
\min _{(z, y) \in A_{\sigma}}\left|u_{\sigma}(z)-u_{\sigma}(y)\right| / 2 & \text { otherwise }
\end{array}\right. \\
\succ_{\sigma}^{x}:=\{\omega \in X: \omega \neq x,\{\omega\}=c(\{\omega, x\}, \sigma)\} \\
\sim_{\sigma}^{x}:=\{\omega \in X: \omega \neq x,\{\omega, x\}=c(\{\omega, x\}, \sigma)\}
\end{gathered}
$$

Using the above, we define: ${ }^{17}$

$$
\varphi(\sigma, x)= \begin{cases}u_{\sigma}(y)-u_{\sigma}(x) \text { for } y \in \sim_{(\sigma, x)}^{x} & \text { if } \exists \theta \neq x \text { s.t. }\{\theta, x\}=c(\{\theta, x\},(\sigma, x)) \\ \max _{\omega \in X}\left[u_{\sigma}(\omega)+\delta_{\sigma}-u_{\sigma}(x)\right] & \text { if }\{x\}=c(\{\omega, x\},(\sigma, x)) \forall \omega \in X \\ \min _{\omega \in \succ_{(\sigma, x)}^{x}}\left[u_{\sigma}(\omega)-\delta_{\sigma}-u_{\sigma}(x)\right] & \text { otherwise }\end{cases}
$$

Finally, define $u_{(\sigma, x)}: X \rightarrow \mathbb{R}$ as:

$$
u_{(\sigma, x)}(y):=u_{\sigma}(y)+\varphi(\sigma, x) \mathbb{1}_{x}(y), \text { for every } y \in X .
$$

\footnotetext{
${ }^{16}$ We write $(\sigma, x)$ to denote the concatenated list that starts with the list $\sigma$ and ends with the element $x$.

${ }^{17}$ The proof that $\varphi(\sigma, x)$ is well defined follows the same lines as the claim.
} 
Note that, for any $\sigma=\left(l_{1}, \ldots, l_{k}\right) \in \mathcal{L}$ and $x \in X$, we have:

$$
u_{\sigma}(x)=u_{\left(l_{1}, \ldots, l_{k-1}\right)}(x)+\varphi\left(l_{1}, \ldots, l_{k}\right) \mathbb{1}_{l_{k}}(x)=u(x)+\sum_{i=1}^{k} \varphi\left(l_{1}, \ldots, l_{i}\right) \mathbb{1}_{l_{i}}(x) .
$$

To conclude the proof we are left to show that $c(S, \sigma)=\underset{x \in S}{\operatorname{argmax}} u_{\sigma}(x)$.

We prove this by induction on the length of $\sigma$. For the base, let $\sigma=(l)$ for some $l \in X$.

case 1: $l \notin S$. By RI:

$$
c(S,(l))=c(S, \emptyset)=\underset{x \in S}{\arg \max } u(x)=\underset{x \in S}{\arg \max } u(x)+\varphi(l) \mathbb{1}_{l}(x),
$$

as we sought.

case 2: $l \in S$. First we show that

$$
c(S,(l)) \subseteq \underset{x \in S}{\operatorname{argmax}} u_{(l)}(x)=\underset{x \in S}{\operatorname{argmax}} u(x)+\varphi(l) \mathbb{1}_{l}(x) .
$$

Take any $x \in c(S,(l))$ and $z \in S \backslash\{x\}$. WARP implies:

$$
x \in c(\{x, z\},(l))
$$

subcase 2.1: $x=l$.

There are three subsubcases to consider:

2.1.1: $\exists y \in X, y \neq x$ such that $\{x, y\}=c(\{x, y\},(x))$.

In this case: $u(x)+\varphi(x)=u\left(y_{0}\right)$ for some $y_{0} \in \sim_{(x)}^{x}$. If $y_{0}=z$ then we are done. So, assume $y_{0} \neq z$. If $\{z\}=c\left(\left\{x, y_{0}, z\right\},(x)\right)$, then by WARP $\{z\}=c(\{x, z\},(x))$, a contradiction to (1). If $\{x\}=c\left(\left\{x, y_{0}, z\right\},(x)\right)$, then by WARP $\{x\}=c\left(\left\{x, y_{0}\right\},(x)\right)$, contradicting $y_{0} \in \sim_{(x)}^{x}$. By a similar argument $\{x, z\} \neq c\left(\left\{x, y_{0}, z\right\},(x)\right)$. We may conclude that $y_{0} \in$ $c\left(\left\{x, y_{0}, z\right\},(x)\right)$. WARP and RI $\left(x \notin\left\{z, y_{0}\right\}\right)$ imply

$$
c\left(\left\{x, y_{0}, z\right\},(x)\right) \cap\left\{y_{0}, z\right\}=c\left(\left\{y_{0}, z\right\},(x)\right)=c\left(\left\{y_{0}, z\right\}, \emptyset\right) .
$$

Thus, $y_{0} \in c\left(\left\{y_{0}, z\right\}, \emptyset\right)$ and therefore $u\left(y_{0}\right) \geq u(z)$.

2.1.2: $\{x\}=c(\{x, y\},(x))$ for all $y \in X$.

Here we have $u(x)+\varphi(x)=\max _{\omega \in X} u(\omega)+\delta_{0}$, so clearly $\max _{\omega \in X} u(\omega)+\delta_{0} \geq u(z)$. 
2.1.3: None of the above.

In this third case: $u(x)+\varphi(x)=u(\underline{\mathrm{z}})-\delta_{0}$, for some $\underline{\mathrm{z}} \in \arg \min _{z \in \succ_{(x)}^{x}} u(z)$. The definition of $\succ_{(x)}^{x}$ and (1) imply $\underline{z} \neq z$. If $x \in c(\{x, z, \underline{z}\},(x))$ then, by WARP, $x \in c(\{x, \underline{z}\},(x))$, a contradiction to $\underline{\mathrm{z}} \in \succ_{(x)}^{x}$. If $z \in c(\{x, z, \underline{\mathrm{z}}\},(x))$ then, by WARP $z \in c(\{x, z\},(x))$ and combined with (1) we obtain $c(\{x, z\},(x))=\{x, z\}$, contradicting subsubcase 2.1.3. Thus, it is only possible that $\{\underline{\mathrm{z}}\}=c(\{x, z, \underline{\mathrm{z}}\},(x))$, and hence $c(\{x, z, \underline{\mathrm{z}}\},(x)) \cap\{z, \underline{\mathrm{z}}\}=\{\underline{\mathrm{z}}\} \neq \emptyset$. Applying WARP and RI $(x \notin\{\underline{z}, z\})$ we find that,

$$
\{\underline{\mathrm{z}}\}=c(\{z, \underline{\mathrm{z}}\},(x))=c(\{z, \underline{\mathrm{z}}\}, \emptyset) .
$$

That is, $u(\underline{\mathrm{z}})>u(z)$. By definition of $\delta_{0}$, it follows that $u(\underline{\mathrm{z}})-\delta_{0}>u(z)$, as we sought.

subcase 2.2: $x \neq l$.

First, if $z \neq l$, then (1), WARP and RI imply $x \in c(\{x, z\},(l))=c(\{x, z\}, \emptyset)$. Hence, $u(x) \geq u(z)$.

If $z=l$, we are to show that $u(x) \geq u(l)+\varphi(l,(l))$.

2.2.1: $\exists y \in X, y \neq l$ such that $\{y, l\}=c(\{y, l\},(l))$.

$u(l)+\varphi(l)=u\left(y_{0}\right)$ for some $y_{0} \in \sim_{(l)}^{l}$. If $x \in \sim_{(l)}^{l}$, then, since $\varphi$ is well defined, $u(x)=u\left(y_{0}\right)$. So, suppose $x \notin \sim_{(l)}^{l}$. Combined with (1), we find that

$$
c(\{x, l\},(l))=\{x\} .
$$

By WARP and (2) $l \notin c\left(\left\{x, l, y_{0}\right\},(l)\right)$. If $\left\{y_{0}\right\}=c\left(\left\{x, l, y_{0}\right\},(l)\right)$ then WARP implies $\left\{y_{0}\right\}=c\left(\left\{l, y_{0}\right\},(l)\right)$ which contradicts $y_{0} \in \sim_{(l)}^{l}$. Thus, $x \in c\left(\left\{x, l, y_{0}\right\},(l)\right)$. We may apply WARP and RI to obtain

$$
x \in c\left(\left\{x, l, y_{0}\right\},(l)\right) \cap\left\{x, y_{0}\right\}=c\left(\left\{x, y_{0}\right\},(l)\right)=c\left(\left\{x, y_{0}\right\}, \emptyset\right) .
$$

It follows that $u(x) \geq u\left(y_{0}\right)$, as we sought.

2.2.2: $\{l\}=c(\{y, l\},(l))$ for every $y \in X$.

Contradicts (1) for $y=x$.

2.2.3: None of the above.

$u(l)+\varphi(l)=u(\underline{\mathrm{z}})-\delta_{0}$ for some $\underline{\mathrm{z}} \in \arg \min _{z \in \succ_{(l)}^{l}} u(z)$. If $u(x)=u(\underline{\mathrm{z}})$ then the result follows because $\delta_{0}>0$. So assume $u(x) \neq u(\underline{\mathrm{z}})$. If $l \in c(\{x, l\},(l))$ then, combined with (1), we have $\{x, l\}=c(\{x, l\},(l))$, contradicting subsubcase 2.2.3. Thus, $\{x\}=c(\{x, l\},(l))$, which implies $x \in \succ_{(l)}^{l} \cdot \underline{\mathrm{z}}$ is $u$-minimal in $\succ_{(l)}^{l}$, therefore $u(x)>u(\underline{\mathrm{z}})$ and clearly $u(x)>u(\underline{\mathrm{z}})-\delta_{0}$.

This completes the first inclusion. For the other inclusion, take $x \in S$ such that $u_{(l)}(x) \geq$ 
$u_{(l)}(y)$ for every $y \in S$. Assume for contradiction $x \notin c(S,(l))$. Take some $z \neq x$ such that $z \in c(S,(l))$. WARP implies that:

$$
c(\{z, x\},(l))=\{z\}
$$

subcase 2.3: $x=l$.

2.3.1: $\exists y \neq x$ such that $c(\{x, y\},(x))=\{x, y\}$.

$u(x)+\varphi(x)=u\left(y_{0}\right)$ for some $y_{0} \in \sim_{(x)}^{x}$. WARP and (3) imply

$x \notin c\left(\left\{z, x, y_{0}\right\},(x)\right)$. Therefore, $c\left(\left\{z, x, y_{0}\right\},(x)\right) \cap\left\{z, y_{0}\right\} \neq \emptyset$. By WARP and RI:

$$
c\left(\left\{z, x, y_{0}\right\},(x)\right) \cap\left\{z, y_{0}\right\}=c\left(\left\{z, y_{0}\right\},(x)\right)=c\left(\left\{z, y_{0}\right\}, \emptyset\right) .
$$

$u\left(y_{0}\right) \geq u(z)$ by assumption and thus, $y_{0} \in c\left(\left\{z, y_{0}\right\}, \emptyset\right)$. It follows that $y_{0} \in c\left(\left\{z, x, y_{0}\right\},(x)\right)$ and hence $c\left(\left\{z, x, y_{0}\right\},(x)\right) \cap\left\{x, y_{0}\right\}=\left\{y_{0}\right\} \neq \emptyset$. By WARP $\left\{y_{0}\right\}=c\left(\left\{x, y_{0}\right\},(x)\right)$, which contradicts $y_{0} \in \sim_{(x)}^{x}$.

2.3.2: $\{x\}=c(\{x, y\},(x)), \forall y \in X$.

Contradicts (3) for $y=z$.

2.3.3: None of the above.

$u(x)+\varphi(x)=u(\underline{\mathrm{z}})-\delta_{0}$ for some $\underline{\mathrm{z}} \in \arg \min _{z \in \succ_{(x)}^{x}} u(z)$. By the assumption, $u(\underline{\mathrm{z}})-\delta_{0}>u(z)$. Thus, $u(\underline{\mathrm{z}})>u(z)$. By $(3), z \in \succ_{(x)}^{x}$, contradicting the $u$-minimality of $\underline{\mathrm{z}}$ in $\succ_{(x)}^{x}$.

subcase 2.4: $x \neq l$.

If $z \neq l$, then by $(3)$ and RI,

$$
\{z\}=c(\{x, z\},(l))=c(\{x, z\}, \emptyset),
$$

hence, $u(z)>u(x)$, a contradiction. So assume $z=l$ and notice that (3) takes the form

$$
\{l\}=c(\{x, l\},(l))
$$

2.4.1: $\exists y \neq l$ such that $c(\{l, y\},(l))=\{l, y\}$.

$u(l)+\varphi(l)=u\left(y_{0}\right)$ for some $y_{0} \in \sim_{(l)}^{l}$. Note that by (4) $y_{0} \neq x$. WARP and (4) imply $x \notin c\left(\left\{l, x, y_{0}\right\},(l)\right)$. If $\{l\}=c\left(\left\{l, x, y_{0}\right\},(l)\right)$ then by WARP $\{l\}=c\left(\left\{l, y_{0}\right\},(l)\right)$, a contradiction to $y_{0} \in \sim_{(l)}^{l}$. Similarly we cannot have $\left\{y_{0}\right\}=c\left(\left\{l, x, y_{0}\right\},(l)\right)$. Thus, $\left\{l, y_{0}\right\}=c\left(\left\{l, x, y_{0}\right\},(l)\right)$. Applying WARP and RI we obtain

$$
\left\{y_{0}\right\}=c\left(\left\{l, x, y_{0}\right\},(l)\right) \cap\left\{x, y_{0}\right\}=c\left(\left\{x, y_{0}\right\},(l)\right)=c\left(\left\{x, y_{0}\right\}, \emptyset\right) .
$$

That is, $u\left(y_{0}\right)>u(x)$ which contradicts the assumption.

2.4.2: $\{l\}=c(\{l, y\},(l))$ for every $y \in X$.

$u(l)+\varphi(l)=\max _{z \in X} u(z)+\delta_{0}$. It follows that $u(l)+\varphi(l)>u(x)$, a contradiction. 
2.4.3: None of the above.

$u(l)+\varphi(l)=u(\underline{\mathrm{z}})-\delta_{0}$ for some $\underline{\mathrm{z}} \in \arg \min _{z \in \succ_{(l)}^{l}} u(z)$. If $l \in c(\{l, x, \underline{\mathrm{z}}\},(l))$ then by WARP $l \in c(\{l, \underline{\mathrm{z}}\},(l))$ contradicting $\underline{\mathrm{z}} \in \succ_{(l)}^{l}$. WARP and (4) imply $x \notin c(\{l, x, \underline{\mathrm{z}}\},(l))$. Thus, we obtain $\{\underline{\mathrm{z}}\}=c(\{l, x, \underline{\mathrm{z}}\},(l))$. Applying WARP and RI,

$$
\{\underline{\mathrm{z}}\}=c(\{l, x, \underline{\mathrm{z}}\},(l)) \cap\{x, \underline{\mathrm{z}}\}=c(\{x, \underline{\mathrm{z}}\},(l))=c(\{x, \underline{\mathrm{z}}\}, \emptyset) .
$$

We may conclude $u(\underline{\mathrm{z}})>u(x)$. By definition of $\delta_{0}, u(\underline{\mathrm{z}})-\delta_{0}>u(x)$. A contradiction.

This completes the induction base. The induction step is proven using identical arguments to the ones used to prove the base along with the induction hypothesis and is therefore omitted.

Proof of Proposition 1. The "if" part is trivial. We show the "only if" part. Let $c$ be a choice correspondence on $\mathcal{C}(X)$ that satisfies WARP, RI and Memory-K for some $K \in \mathbb{N}$. Establish $u: X \rightarrow \mathbb{R}$ exactly as in the proof of Theorem 1 such that:

$$
c(S, \emptyset)=\underset{x \in S}{\arg \max } u(x) \text { for every } S \in \Omega_{X} .
$$

Take any $x, y \in X$ and $\sigma \in \mathcal{L}$ and let $z \in X$ be such that $z \neq x$ and $z \neq y$. Then:

$$
c(\{x, y\}, \sigma)=c(\{x, y\},(\sigma, \underbrace{z, . ., z}_{K}))=c(\{x, y\},(\underbrace{z, . ., z}_{K}))=c(\{x, y\}, \emptyset)
$$

where the first and last equalities are due to RI while the second due to Memory-K. Since $x, y$ were arbitrary elements in $X$, and $\sigma$ an arbitrary list, a standard argument based on WARP shows that for any $S \in \Omega_{X}$ and $\sigma \in \mathcal{L}$ :

$$
c(S, \sigma)=c(S, \emptyset)
$$

Proof of Proposition 2. Once again we only prove the "only if" part. Let $c$ be a choice correspondence on $\mathcal{C}(X)$ that satisfies WARP, RI and WSQB. Following the proof of Theorem 1 we obtain the representation. We only need to show that $\varphi \geq 0$. Take any list $\bar{\sigma} \in L$. Wlog we can write $\bar{\sigma}=(\sigma, x)$ for some $x \in X$. Following the definition of $\varphi$, we have three cases to consider:

1. $\varphi(\sigma, x)=u_{\sigma}(y)-u_{\sigma}(x)$ for $y \in \sim_{(\sigma, x)}^{x}$. If $\varphi(\sigma, x)<0$ then $u_{\sigma}(y)-u_{\sigma}(x)<0$. Thus $\{x\}=c(\{x, y\}, \sigma)$. If $y \in c(\{x, y\},(\sigma, x))$ then by WSQB $y \in c(\{x, y\}, \sigma)$, a contradiction. So, it can only be that $\{x\}=c(\{x, y\},(\sigma, x))$, contradicting $y \in \sim_{(\sigma, x)}^{x}$. 
2. $\varphi(\sigma, x)=\max _{\omega \in X}\left[u_{\sigma}(\omega)+\delta_{\sigma}-u_{\sigma}(x)\right]$. We may conclude $\varphi(\sigma)>u_{\sigma}(\bar{\omega})-u_{\sigma}(x) \geq 0$. where $\bar{\omega} \in \underset{\omega \in X}{\operatorname{argmax}}\left[u_{\sigma}(\omega)+\delta_{\sigma}-u_{\sigma}(x)\right]$.

3. $\varphi(\sigma, x)=\min _{\omega \in \succ_{(\sigma, x)}^{x}}\left[u_{\sigma}(\omega)+\delta_{\sigma}-u_{\sigma}(x)\right]$. There are two subcases to consider:

(i) $A_{\sigma}=\emptyset$. In this case $u_{\sigma}(\omega)=u_{\sigma}(x), \forall \omega \in X \Rightarrow x \in c(\{x, w\}, \sigma), \forall \omega \in X$. By WSQB $x \in c(\{x, w\},(\sigma, x)), \forall \omega \in X$, a contradiction to case 3 .

(ii) $A_{\sigma} \neq \emptyset$. Assume $\varphi(\sigma, x)<0$. By definition of $\varphi, u_{\sigma}(\underline{\mathrm{z}})-\delta_{\sigma}-u_{\sigma}(x)<0$, where $\underline{\mathrm{z}} \in \min _{\omega \in \succ_{(\sigma, x)}^{x}}\left[u_{\sigma}(\omega)+\delta_{\sigma}-u_{\sigma}(x)\right]$. If $u_{\sigma}(\underline{\mathrm{z}})>u_{\sigma}(x)$ then $u_{\sigma}(\underline{\mathrm{z}})-u_{\sigma}(x)<\delta_{\sigma}$, which contradicts the definition of $\delta_{\sigma}$. If $u_{\sigma}(\underline{\mathrm{z}})<u_{\sigma}(x)$ then $\{x\}=c(\{x, \underline{\mathrm{z}}\}, \sigma)$. If $\underline{\mathrm{z}} \in c(\{x, \underline{\mathrm{z}}\},(\sigma, x))$ then by WSQB $\underline{z} \in c(\{x, \underline{z}\}, \sigma)$, a contradiction. Thus it must be the case that $\{x\}=$ $c(\{x, \underline{\mathrm{z}}\},(\sigma, x))$ which contradicts $\underline{\mathrm{z}} \in \succ_{(\sigma, x)}^{x}$. Finally, if $u_{\sigma}(\underline{\mathrm{z}})=u_{\sigma}(x)$, then $x \in c(\{x, \underline{\mathrm{z}}\}, \sigma)$. By WSQB $x \in c(\{x, \underline{\mathrm{z}}\},(\sigma, x))$, once again contradicting $\underline{\mathrm{z}} \in \succ_{(\sigma, x)}^{x}$.

Proof of Proposition 3. Once again we only prove the "only if" part. Let $c$ be a choice correspondence on $\mathcal{C}(X)$ that satisfies WARP, RI and MSQB. Following the proof of Theorem 1 we obtain the representation. We only need to show that $\varphi>0$. Take any list $\bar{\sigma} \in L$ and suppose wlog we can write $\bar{\sigma}=(\sigma, x)$ for some $x \in X$. Following the definition of $\varphi$, we have three cases to consider:

1. $\varphi(\sigma, x)=u_{\sigma}(y)-u_{\sigma}(x)$ for $y \in \sim_{(\sigma, x)}^{x}$. If $\varphi(\sigma, x) \leq 0$ then $u_{\sigma}(y)-u_{\sigma}(x) \leq 0$. Thus $x \in c(\{x, y\}, \sigma)$. By MSQB $\{x\}=c(\{x, y\},(\sigma, x))$, contradicting $y \in \sim_{(\sigma, x)}^{x}$.

2. $\varphi(\sigma, x)=\max _{\omega \in X}\left[u_{\sigma}(\omega)+\delta_{\sigma}-u_{\sigma}(x)\right]$. We may conclude $\varphi(\sigma)>u_{\sigma}(\bar{\omega})-u_{\sigma}(x) \geq 0$. where $\bar{\omega} \in \underset{\omega \in X}{\operatorname{argmax}}\left[u_{\sigma}(\omega)+\delta_{\sigma}-u_{\sigma}(x)\right]$.

3. $\varphi(\sigma, x)=\min _{\omega \in \succ_{(\sigma, x)}^{x}}\left[u_{\sigma}(\omega)+\delta_{\sigma}-u_{\sigma}(x)\right]$. There are two subcases to consider:

(i) $A_{\sigma}=\emptyset$. In this case $u_{\sigma}(\omega)=u_{\sigma}(x), \forall \omega \in X \Rightarrow x \in c(\{x, w\}, \sigma), \forall \omega \in X$. By MSQB $\{x\}=c(\{x, w\},(\sigma, x)), \forall \omega \in X$ a contradiction to case 3 .

(ii) $A_{\sigma} \neq \emptyset$. Assume $\varphi(\sigma, x) \leq 0$. By definition of $\varphi, u_{\sigma}(\underline{\mathrm{z}})-\delta_{\sigma}-u_{\sigma}(x) \leq 0$, where $\underline{\mathrm{z}} \in$ $\min _{\omega \in \succ_{(\sigma, x)}^{x}}\left[u_{\sigma}(\omega)+\delta_{\sigma}-u_{\sigma}(x)\right]$. If $u_{\sigma}(\underline{\mathrm{z}})>u_{\sigma}(x)$ then $u_{\sigma}(\underline{\mathrm{z}})-u_{\sigma}(x) \leq \delta_{\sigma}$, which contradicts the definition of $\delta_{\sigma}$. If $u_{\sigma}(\underline{\mathrm{z}}) \leq u_{\sigma}(x)$ then $x \in c(\{x, \underline{\mathrm{z}}\}, \sigma)$. By MSQB $\{x\}=c(\{x, \underline{\mathrm{z}}\},(\sigma, x))$, which contradicts $\underline{\mathrm{z}} \in \succ_{(\sigma, x)}^{x}$. 


\section{References}

Abel, A. B. (1990): "Asset Prices under Habit Formation and Catching up with the Joneses," American Economic Review, 80(2), 38-42.

Becker, G. S. And K. M. Murphy (1988): "A Theory of Rational Addiction," Journal of Political Economy, 96(4), 675-700.

Bloemer, J. M. And H. D. Kasper (1995): "The complex relationship between consumer satisfaction and brand loyalty," Journal of Economic Psychology, 16(2), 311-329.

Boldrin, M., L. J. Christiano, and J. D. Fisher (1997): "Habit Persistence and Asset Returns in an Exchange Economy," Macroeconomic Dynamics, 1(2), 312-332.

Choi, J. J., D. Laibson, B. C. Madrian, and A. Metrick (2004): "For better or for worse: Default effects and 401(k) savings behavior," in Perspectives on the Economics of Aging, ed. by D. Wise, University of Chicago Press.

Crandall, C. S. (1985): "The Liking of Foods as a Result of Exposure: Eating Doughnuts in Alaska," Journal of Social Psychology, 125(2), 187-194.

Dean, M. (2008): "Status Quo Bias in Large and Small Choice Sets," Working Paper.

Erdem, T. And M. P. Keane (1996): "Decision-Making under Uncertainty: Capturing Dynamic Brand Choice Processes in Turbulent Consumer Goods Markets," Marketing Science, 15(1), 1-20.

Gilboa, I. And A. Pazgal (2001): "Cumulative Discrete Choice," Marketing Letters, 12(2), 119-130.

Grossman, M., F. J. Chaloupka, and R. T. Anderson (1998): "A Survey of Economic Models of Addictive Behavior," Journal of Drug Issues, 28(3), 631-643.

Grne-Yanoff, T. And S. O. Hansson (2009): "Preference Change: An Introduction," in Preference Change, ed. by T. Grne-Yanoff and S. O. Hansson, Springer.

Jeuland, A. P. (1978): "Brand Preference Over Time: A Partially Deterministic Operationalization of the Notion of Variety Seeking," in Research Frontiers in Marketing: Dialogues and Directions, ed. by S. C. Jain, American Marketing Association.

Johnson, E. J. and D. G. Goldstein (2003): "Do Defaults Save Lives?" Science, 302, 1338-1339.

KAHN, B. E. (1995): "Consumer variety-seeking among goods and services: An integrative review," Journal of Retailing and Consumer Services, 2(3), 139-148. 
Kahn, B. E., M. Kalwani, And D. G. Morrison (1986): "Measuring Variety-Seeking and Reinforcement Behaviors Using Panel Data," Journal of Marketing Research, 23(2), 89-100.

Kempf, A. And S. Ruenzi (2006): "Status Quo Bias and the Number of Alternatives: An Empirical Illustration from the Mutual Fund Industry," Journal of Behavioral Finance, $7(4), 204-213$.

Klemperer, P. (1987): "Markets with Consumer Switching Costs," The Quarterly Journal of Economics, 102(2), 375-394.

Knetsch, J. L. (1989): "The Endowment Effect and Evidence of Nonreversible Indifference Curves," American Economic Review, 79(5), 1277-1284.

Knetsch, J. L. And W. K. Wong (2009): "The endowment effect and the reference state: Evidence and manipulations," Journal of Economic Behavior and Organization, $71(2), 407-413$.

Madrian, B. C. And D. F. Shea (2001): "The Power of Suggestion: Inertia in 401(k) Participation and Savings Behavior," Quarterly Journal of Economics, 116(4), 1149 1187.

Masatlioglu, Y. And E. A. OK (2005): "Rational Choice with Status Quo Bias," Journal of Economic Thoery, 121(1), 1-29.

- (2014): "A Canonical Model of Choice with Initial Endowments," The Review of Economic Studies, 81(2), 851-883.

Meyer, R., T. Erdem, F. Feinberg, I. Gilboa, W. Hutchinson, A. Krishna, S. Lippman, C. Mela, A. Pazgal, D. Prelec, and J. H. Steckel (1997): "Dynamic Influences on Individual Choice Behavior," Marketing Letters, 8(3), 349-360.

Meyer, R. J. And A. Sathi (1985): "A Multiattribute Model of Consumer Choice During Product Learning," Marketing Science, 4(1), 41-61.

Ortoleva, P. (2010): "Status quo bias, multiple priors and uncertainty aversion," Games and Economic Behavior, 69(2), 411-424.

Pliner, P. (1982): "The Effects of Mere Exposure on Liking for Edible Substances," Appetite, 3(3), 283-290.

Riella, G. And R. Teper (2014): "Probabilistic dominance and status quo bias," Games and Economic Behavior, 87, 288-304. 
Rolls, B. J., E. Rowe, E. Rolls, B. Kingston, A. Megson, And R. Gunary (1981): "Variety in a meal enhances food intake in man," Physiology and Behavior, 26(2), 215-221.

Rozen, K. (2010): "Foundations of Intrinsic Habit Formation," Econometrica, 78(4), $1341-1373$.

Salant, Y. And A. Rubinstein (2008): "(A,f): Choice with Frames," Review of Economic Studies, 75(4), 1287-1296.

Samuelson, W. And R. Zeckhauser (1988): "Status Quo Bias in Decision Making," Journal of Risk and Uncertainty, 1(1), 7-59.

Stevenson, R. J. And M. R. Yeomans (1995): "Does exposure enhance liking for the chilli burn?" Appetite, 24(2), 107-120.

Thaler, R. (1980): "Toward a positive theory of consumer choice," Journal of Economic Behavior and Organization, 1(1), 39-60.

Zajonc, R. B. (1968): "Attitudinal Effects of Mere Exposure," Journal of Personality and Social Psychology, 9(2 Pt. 2), 1-27. 